\title{
Imaging of Inflammation by PET, Conventional Scintigraphy, and Other Imaging Techniques*
}

\author{
Martin Gotthardt ${ }^{1,2}$, Chantal P. Bleeker-Rovers ${ }^{2,3}$, Otto C. Boerman ${ }^{1,2}$, and Wim J.G. Oyen ${ }^{1,2}$ \\ ${ }^{I}$ Department of Nuclear Medicine, Radboud University Nijmegen Medical Centre, Nijmegen, The Netherlands; ${ }^{2}$ Nijmegen Institute for \\ Infection, Inflammation and Immunity, Radboud University Nijmegen Medical Centre, Nijmegen, The Netherlands; and ${ }^{3}$ Department \\ of Internal Medicine, Radboud University Nijmegen Medical Centre, Nijmegen, The Netherlands
}

\begin{abstract}
Nuclear medicine imaging procedures play an important role in the assessment of inflammatory diseases. With the advent of 3dimensional anatomic imaging, there has been a tendency to replace traditional planar scintigraphy by CT or MRI. Furthermore, scintigraphic techniques may have to be combined with other imaging modalities to achieve high sensitivity and specificity, and some may require time-consuming labeling procedures. On the other hand, new developments such as combined SPECT/CT increase the diagnostic power of scintigraphy. Also, the advent of PET had a considerable impact on the use of nuclear medicine imaging techniques. In this review, we aim to provide nuclear medicine specialists and clinicians with the relevant information on rational and efficient use of nuclear medicine imaging techniques in the assessment of patients with osteomyelitis, infected vascular prostheses, metastatic infectious disease, rheumatoid arthritis, vasculitis, inflammatory bowel disease, sarcoidosis, and fever of unknown origin.
\end{abstract}

Key Words: infectious disease; PET; SPECT; inflammation; radionuclide imaging

J Nucl Med Technol 2013; 41:157-169

DOI: 10.2967/jnumed.110.076232

\section{A}

part from clinical examination, the diagnosis of inflammatory diseases usually requires laboratory tests and imaging procedures. The approach to diagnosing a specific inflammatory disease depends on the type of suspected disease and the clinical presentation. Therefore, the context in which imaging procedures are used varies considerably. For many inflammatory diseases, no unequivocal guidelines for the use of imaging procedures exist, but in some cases there is sufficient evidence from the literature to advise on im-

\footnotetext{
Received Jun. 8, 2010; revision accepted Oct. 12, 2010.

For correspondence or reprints contact: Martin Gotthardt, Department of Nuclear Medicine, Radboud University, Nijmegen Medical Centre, P.O. Box 91016500 HB Nijmegen, The Netherlands.

E-mail: m.gotthardt@nucmed.umcn.nl

*NOTE: FOR CE CREDIT, YOU CAN ACCESS THIS ACTIVITY THROUGH THE SNMMI WEB SITE (http://www.snmmi.org/ce_online) THROUGH SEPTEMBER 2015. PARTICIPANTS WHO HAVE ALREADY TAKEN THE EXAM USING JNM AND PASSED CANNOT RETAKE THE EXAM.

Published online Aug. 8, 2013.

COPYRIGHT @ 2013 by the Society of Nuclear Medicine and Molecular Imaging, Inc.
}

aging procedures for optimal diagnosis or follow-up. In other cases, local factors and specific expertise with a certain diagnostic algorithm may play an important role. Here, we provide a concise summary of the most important and widespread nuclear medicine imaging techniques in inflammatory diseases for clinicians and nuclear medicine specialists. This paper focuses on imaging in important infectious diseases such as osteomyelitis, vascular graft infection, and metastatic infectious disease; imaging of noninfectious inflammatory diseases such as rheumatoid arthritis (RA), vasculitis, inflammatory bowel disease (IBD), and sarcoidosis; and fever of unknown origin (FUO). Imaging of other inflammatory diseases such as infection of joint prostheses has been specifically covered by recent reviews and is therefore not the subject of this paper.

\section{IMAGING TECHNIQUES}

Imaging techniques for the detection of inflammation include ultrasonography, CT, MRI, endoscopic techniques, PET, planar scintigraphy, and SPECT. All these techniques have specific advantages and disadvantages. To better understand the roles of these modalities in relation to the nuclear medicine techniques, it is important to be aware of their respective advantages and disadvantages.

Ultrasonography is widely available, quick, inexpensive, and not associated with radiation exposure. It has an excellent spatial resolution reaching below $1 \mathrm{~mm}$ and can be used to obtain functional information to a limited extent (e.g., blood flow by Doppler ultrasonography). The disadvantages are that the results are highly operator-dependent, penetration and reflection of the sound waves in tissue may be hindered by gas (bowel) or dense structures (bone), and structures deep within the body may be difficult to visualize because the image quality suffers from the longer wavelengths used for deep imaging.

CT is highly reproducible, has an excellent spatial resolution, and, although more expensive than ultrasonography, is still relatively inexpensive. The disadvantages are exposure of the patient to radiation and the lack of functional information. Furthermore, the use of contrast medium to enhance image contrast may be limited or impossible in patients with impaired renal function or previous allergic reactions. 
MRI is also characterized by a high resolution, has some potential to obtain functional information, and causes no radiation exposure. It has also become widely available but is prone to movement artifacts because of the relatively long examination time. Because of the long imaging times and the considerable exposure to noise, MRI is not convenient for patients. Furthermore, there are limitations to the scanning of patients with pacemakers, implants, and other devices, and the procedure is relatively expensive.

Although PET and SPECT have a limited spatial resolution (PET down to 3-5 mm; SPECT down to 8-10 $\mathrm{mm}$ ), their asset is high contrast resolution, offering functional and molecular information with high sensitivities in the nano- or picomolar range (PET $>$ SPECT). The radiation exposure is mostly low but, dependent on the radiotracer used, may reach the radiation exposure of an abdominal CT scan.

\section{RADIOPHARMACEUTICALS}

Radiopharmaceuticals for imaging of inflammatory disease should be highly sensitive (apart from having general characteristics such as easy preparation, wide availability, and low cost). In addition, in an ideal situation radiopharmaceuticals would also be able to distinguish between infectious and sterile inflammation. A wide variety of radiotracers has been tested for imaging of inflammation to achieve the desired characteristics. However, only a few agents are currently in general use for inflammation imaging. These include ${ }^{18} \mathrm{~F}-\mathrm{FDG}$, autologous white blood cells (WBCs [leukocytes]) labeled with ${ }^{99 \mathrm{~m} T c}$ or ${ }^{111} \mathrm{In},{ }^{99 \mathrm{~m}} \mathrm{Tc}$-labeled bisphosphonates such as methylene diphosphonate or hydroxymethylene diphosphonate, ${ }^{67} \mathrm{Ga}$-citrate, ${ }^{99 \mathrm{~m}} \mathrm{Tc}$-labeled nanocolloids, and ${ }^{99 \mathrm{~m} T c-}$ or ${ }^{111} \mathrm{In}$-labeled proteins, such as IgG or albumin. None is specific for inflammation, and none offers the possibility of directly distinguishing sterile from septic inflammation. The accumulation of these agents in inflamed tissue is based on different mechanisms. The first mechanism is uptake into inflamed tissue as a result of increased metabolism, either of inflammatory cells $\left({ }^{18} \mathrm{~F}-\mathrm{FDG}\right.$, as a glucose analog reflecting the energy demand of inflammatory cells) or of tissue-specific cells with increased activity as a reaction to inflammation ${ }^{99 \mathrm{~m}} \mathrm{Tc}-$ hydroxymethylene diphosphonate and ${ }^{99 \mathrm{~m}} \mathrm{Tc}$-methylene diphosphonate, reflecting the activity of osteoblasts as the active response of bone to inflammation). The second mechanism is unspecific accumulation in the site of inflammation as a result of increased blood flow and enhanced vascular permeability (albumin, $\mathrm{IgG})$. In the case of labeled activated leukocytes, the uptake mechanism is specific migration to the site of inflammation. Finally, ${ }^{67} \mathrm{Ga}$ binds to transferrin, with the complex being extravasated at sites of inflammation because of increased vascular permeability and then being transferred to locally present lactoferrin. Most radiolabeled agents would accumulate at sites of infection if the local blood flow and the vascular permeability were increased, but local binding also plays a role as exemplified by ${ }^{67} \mathrm{Ga}$-citrate. Bisphosphonates are another example of several mechanisms playing a role in the accumulation of a radiotracer. Bisphosphonates show increased uptake at sites of inflammation on early images taken directly after injection of the radiopharmaceutical (arterial phase, as a result of increased blood flow) and a few minutes after injection (blood-pool phase, as a result of increased vascular permeability in combination with increased blood flow) and on the delayed images several hours after injection (static phase, as a result of increased bone metabolism reflecting osteoblastic activity at sites of inflammation) (Table 1).

\section{IMAGING OF INFECTIOUS DISEASES}

\section{Osteomyelitis}

Three-phase bone scanning with ${ }^{99 \mathrm{~m}} \mathrm{Tc}$-hydroxymethylene diphosphonate or ${ }^{99 \mathrm{~m}} \mathrm{Tc}-$ methylene diphosphonate has long been used as the standard method for the detection of osteomyelitis. Bone scintigraphy has a high sensitivity exceeding $80 \%$ and a limited specificity reaching up to $50 \%$ (Fig. 1) $(1,2)$. The limited specificity can be explained by uptake of the radiopharmaceutical at all sites of increased bone metabolism irrespective of the underlying cause. However, if combined SPECT/CT is performed, the specificity increases considerably to above $80 \%$ (3). As an alternative to bone scanning, WBC imaging has been proposed. Because of physiologic uptake into bone marrow, both sensitivity and specificity may be impaired (4). Therefore, combined imaging of inflammation with WBC and nanocolloids for bone marrow has been evaluated. Because, in osteomyelitis, bone marrow is replaced by the infectious process, bone marrow imaging will be negative whereas WBC scanning in the same location will be positive. This combined imaging approach will result in an excellent sensitivity and specificity above $90 \%$ (5). Also, the combination of WBC and bone SPECT results in high sensitivities and specificities above $90 \%$. This combination has been shown to be superior to MRI and CT in postoperative patients with osteomyelitis in the mastoid region (6). However, in spondylitis and spondylodiscitis, WBC imaging results in low sensitivities around $50 \%$, which has been hypothesized to reflect the inability of leukocytes to migrate into the encapsulated infection (6). These photopenic lesions are not specific for infection and, together with the physiologic uptake of WBCs into the bone marrow, hamper accurate detection of spinal infection (4).

${ }^{18}$ F-FDG PET has also been evaluated in patients with osteomyelitis. PET has been shown to offer an excellent sensitivity, normally reaching or exceeding $95 \%$, with high specificities above $87 \%(7,8)$. In spondylitis and spondylodiscitis, PET combines high sensitivity with high specificity (9-11). Gratz et al. showed that PET was superior to MRI (9). De Winter et al. were able to demonstrate that the specificity for spinal infection dropped only if patients had undergone surgery less than 6 mo before PET (specificity 75\%) and if osteosynthetic material was present (specificity 65\%) (11). However, because MRI may not be an option in patients with metallic implants in situ, PET currently is the most sensitive imaging modality in the evaluation of such patients (11). Furthermore, in cases of severe degenerative disk disease 


\begin{tabular}{|c|c|}
\hline Radiopharmaceutical & Mechanism \\
\hline $18 \mathrm{~F}-\mathrm{FDG}$ & Macrophages (metabolically active cells), leukocytes \\
\hline $\begin{array}{l}\text { 99mTc/ }{ }^{111} \text { In-labeled autologous WBCs } \\
\text { (leukocytes) }\end{array}$ & Active migration into sites of inflammation \\
\hline 99mTc-labeled bisphosphonates & $\begin{array}{l}\text { Uptake in sites of increased perfusion and extravasation (early phase) and } \\
\text { increased bone formation (late phase) }\end{array}$ \\
\hline${ }^{67} \mathrm{Ga}$-citrate & $\begin{array}{l}\text { Increased perfusion, extravasation due to increased vessel permeability, locally } \\
\text { binding to lactoferrin }\end{array}$ \\
\hline 99mTc-labeled nanocolloids & Uptake in macrophages (inflammation, bone marrow, liver, spleen) \\
\hline 99mTc/111In-labeled proteins (IgG, albumin) & Extravasation (increased perfusion and vessel permeability) \\
\hline
\end{tabular}

with edemalike changes in the endplates and the adjacent discs, MRI can give false-positive results (12).

In diabetic patients, PET with ${ }^{18} \mathrm{~F}-\mathrm{FDG}$ may be at a disadvantage because peripheral insulin resistance might cause decreased uptake at the site of inflammation (13). In diabetic-foot patients with clinically suspected osteomyelitis, ${ }^{18}$ F-FDG PET showed an only slightly lower sensitivity than MRI for the detection of osteomyelitis but a higher specificity (81\% and $93 \%$ as compared with $91 \%$ and $78 \%$, respectively) (14), whereas in diabetic-foot patients without clinical suspicion of osteomyelitis, MRI was clearly superior (13). On the basis of these results, PET seems to be complementary to MRI and may be used as an alternative to MRI after plain radiography as a screening method (14).

In routine clinical practice, several factors have to be considered for efficient imaging of suspected osteomyelitis. These factors include, besides the sensitive and specific detection of disease, the time needed to complete the procedure, ease for the patient, and radiation exposure. MRI has been shown to be sensitive for detection of osteomyelitis, the typical sign of osteomyelitis on MR images being bone edema, possibly associated with destruction of vertebral endplates in later stages of spondylodiscitis (12). Because in most cases osteomyelitis will be limited to a specific bone or adjacent bones of a specific area, whole-body imaging is not necessary. MRI has therefore started to replace bone scintigraphy as the imaging procedure of choice in osteomyelitis, and the comparably inconvenient combination of WBC scanning, bone marrow scanning, and bone scanning is less frequently performed (12).

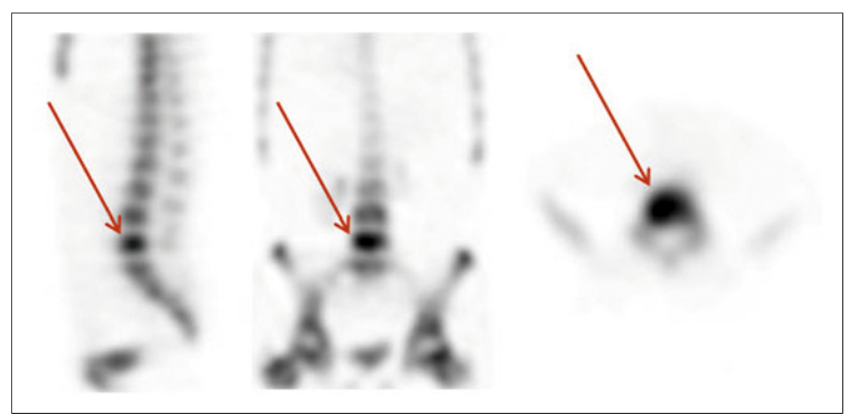

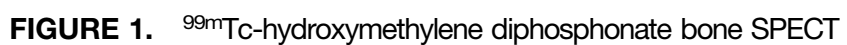
scan of patient with osteomyelitis of fifth lumbar vertebra (arrows).
In the case of suspected osteomyelitis in children, dissemination with foci in several bones has to be kept in mind. These foci can easily be detected by bone scintigraphy or ${ }^{18}$ F-FDG PET. However, with the advent of whole-body MRI, MRI is at an advantage because it does not expose children to radiation. At this time, whole-body MRI is becoming more widely available, and shortcomings (limited number of sequences, extra effort required for imaging in axial direction for higher sensitivity, image realignment if moving table-top is not available) are largely overcome $(15,16)$. Therefore, to avoid radiation exposure, pediatricians tend to perform whole-body MRI rather than ${ }^{18} \mathrm{~F}-\mathrm{FDG}$ PET or bone scanning in cases of suspected osteomyelitis (16).

Although MRI of bone infection may be highly accurate and convenient for physicians and patients, nuclear medicine imaging may be equivalent or even offer some advantages. In patients with pacemakers or other metallic implants, PET is an excellent alternative to MRI. Furthermore, combined SPECT/CT increases the specificity of bone scanning, rendering this technique an alternative if PET is not available. Highly innovative SPECT reconstruction algorithms increase image quality and potentially also the sensitivity of SPECT (17). Therefore, the competition between MRI and PET/CT or SPECT/CT may endure, and thoroughly planned prospective controlled clinical trials have to be performed to answer the question of which imaging modality is most efficient for the detection of osteomyelitis and spondylitis or spondylodiscitis. At this time, for imaging of peripheral osteomyelitis as well as spondylitis or spondylodiscitis, MRI or ${ }^{18}$ F-FDG PET are advised. If they not available, SPECT may be an alternative. Also, in patients with claustrophobia, bone scanning or PET may be used instead of MRI.

\section{Infected Vascular Grafts}

Early diagnosis of vascular graft infection is of the utmost importance in patient management. The rate of infection of vascular grafts ranges from $0.5 \%$ to $5 \%$ and is associated with a high risk of morbidity (such as limb loss) and mortality. Because bacterial eradication is rarely possible, surgical intervention-removal of the infected graftis required $(18,19)$. 
For the diagnosis of vascular graft infection, not only a high sensitivity but also a high specificity is required in view of the considerable consequences of a false-positive diagnosis. On CT scans, air bubbles can be detected around the infected graft in about half the cases but are highly unspecific, because in 50\% of grafts these bubbles are present for weeks or even months after surgery (19). Also, it is difficult to differentiate between acute infection, hematoma, and lymphocele on CT scans, and CT is often false-negative in chronic low-grade infections (19). In comparison to CT, PET/ $\mathrm{CT}$ has shown superior diagnostic performance. PET has shown a sensitivity exceeding 90\%, in comparison to $64 \%$ for CT (20). Although the specificity was not sufficient, reaching only $64 \%$, it exceeded $90 \%$ with the adoption of clear criteria for image interpretation (only focal abnormal uptake interpreted as positive for infection) (20). These data have been confirmed recently by another study (Fig. 2) $(21,22)$. The linear mildly to moderately increased uptake around vascular grafts seems to be attributable to a chronic aseptic inflammation in the synthetic graft material (19). It appears that inhomogeneously increased uptake should be interpreted as a nondiagnostic PET scan, because in this group of patients the sensitivity and specificity were only $73 \%$ and $86 \%$, respectively (22). In another study, the value of combined PET/ CT could be demonstrated, allowing exact determination of the location of the infectious focus, thus resulting in high positive and negative predictive values of $88 \%$ and $96 \%$, respectively. Imaging of vascular graft infection by labeled WBCs suffers from a chance of false-positive results caused by the inability to exactly localize the site of inflammation and a lower sensitivity than PET $(19,23)$.

Therefore, as long as the exact role of MRI remains to be established in the diagnosis of vascular graft infection, PET/CT seems to have the higher sensitivity. The criteria as defined by Fukuchi et al. and Spacek et al. should be used in the assessment of PET scans for vascular graft infection to guarantee sufficient specificity $(20,22)$. In the group of nondiagnostic PET scans (22), an alternative imaging modality with high specificity should be used. WBC scanning appears to be most appropriate for this task. Although it seems as if PET, being superior to CT (24), may be the imaging procedure of choice, one cannot finally conclude at this time-based on the available literature-that PET is also superior to WBC scanning. However, in view of the high sensitivity of PET and the fact that PET is more convenient for patients and health care professionals alike, we would recommend PET/CT first, followed by WBC imaging for the nondiagnostic group. In addition, PET should be performed at least 2 mo after surgery to avoid false-positive results. Because most vascular graft infections are diagnosed late (at least $4 \mathrm{mo}$ ) after surgery, this limitation plays a minor role (19).

\section{Detection of Metastatic Infectious Disease}

Timely detection of metastatic infectious foci in Grampositive bacteremia is crucial, because these foci often re- quire prolonged antibiotic treatment or drainage. Diagnosis of metastatic infectious foci is difficult because localizing symptoms are often absent. ${ }^{18} \mathrm{~F}$-FDG PET has been assessed in the detection of metastatic infectious foci (24). In the 40 patients evaluated in this retrospective study, metastatic complications were diagnosed in $75 \%$. It could be demonstrated that, although a median number of 4 diagnostic procedures had been performed before PET, PET identified clinically relevant new foci in $45 \%$ of cases. The positive and negative predictive values of PET were $91 \%$ and $99 \%$, respectively (24). In a recent prospective study, ${ }^{18} \mathrm{~F}-\mathrm{FDG}$ PET was performed on 115 nonneutropenic patients with Grampositive bacteremia and at least 1 risk factor for developing infectious complications. The results were compared with 230 matched historical controls in whom no ${ }^{18}$ F-FDG PET had been performed. Significantly more patients were diagnosed with metastatic foci in the study group $(67.8 \%$ vs. $35.7 \%$ ). The sensitivity, specificity, and negative and positive predictive values of ${ }^{18} \mathrm{~F}$-FDG PET/CT were $100 \%, 87 \%$, $100 \%$, and $89 \%$, respectively. Overall mortality after 6 mo decreased from $32 \%$ to $19 \%$ in the ${ }^{18} \mathrm{~F}$-FDG PET/CT group $(P=0.014)$ because of a decrease in relapse rate $(25)$.

Therefore, ${ }^{18} \mathrm{~F}$-FDG PET is a valuable imaging technique for the detection of metastatic infectious foci and can detect foci even when other imaging procedures are negative. Because of its high sensitivity, PET should be performed early in all patients with high-risk Gram-positive bacteremia and should be considered in patients with suspected disseminated infection (Fig. 3).

\section{IMAGING OF NONINFECTIOUS INFLAMMATORY DISEASE \\ RA}

RA is an autoimmune chronic disease destroying joints and eventually leading to permanent disability. Because the early signs of RA are not necessarily specific, early diagnosis may be challenging. Moreover, because the presentation of the disease may vary considerably, a defined approach needs to be used to evaluate treatment response and disease activity. To integrate all the different aspects of disease activity and response monitoring, different sets of criteria have been developed, among which those of the American College of Rheumatologists are well suited for response assessment whereas the widely used DAS28 criteria aim to define absolute measures of disease activity, allowing its continuous monitoring.

Monitoring the effects of anti-RA drug treatment for optimal patient therapy has become even more important through the individualized use of disease-modifying antirheumatic drugs, such as methotrexate, sulfasalazine, leflunomide, and, more recently, biologicals (26). For optimization of therapy to achieve low disease activity in patients with a longstanding history of RA, or to achieve remission in newly diagnosed patients, it is important to sensitively measure response to treatment, and such a measurement cannot always be easily based on clinical criteria (26). Another 


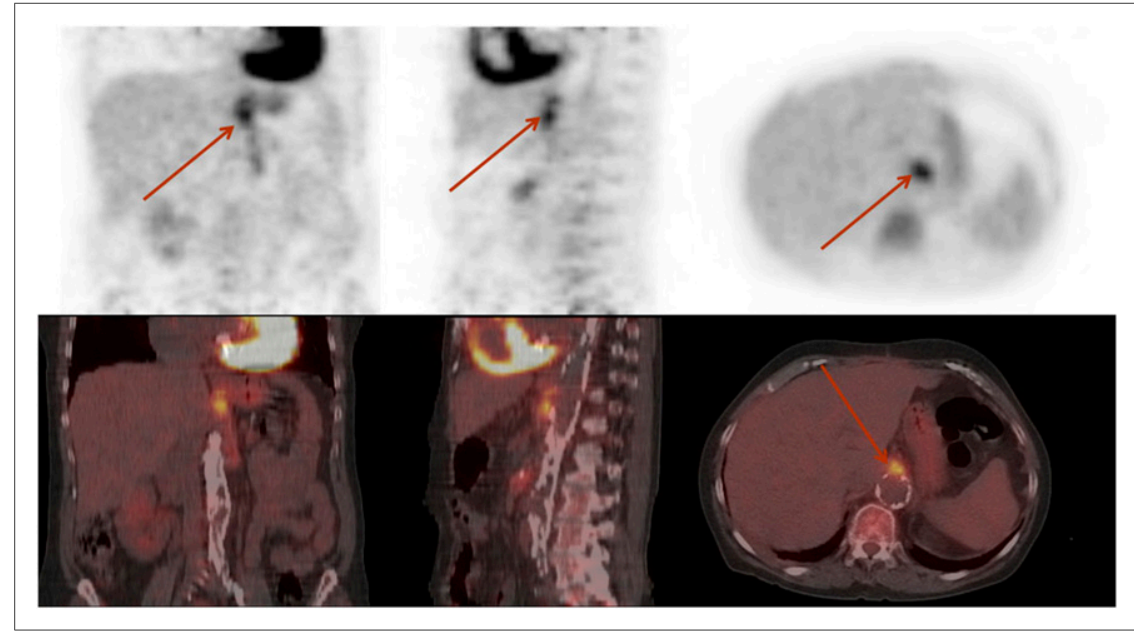

FIGURE 2. ${ }^{18} \mathrm{~F}-\mathrm{FDG}$ PET/CT scan of patient with proven Escherichia coli infection of vascular graft. Focal, intense uptake of ${ }^{18} \mathrm{~F}-\mathrm{FDG}$ (arrows) is sign of infection, in comparison with more diffusely increased physiologic uptake along graft. aspect at an early time point is to determine the optimal moment to start treatment before irreversible joint damage has occurred. Finally, there is also a need to stratify patients into those with a low risk or a high risk of rapid disease progression and severe joint destruction.

Imaging procedures may help to overcome the challenges of diagnosis and therapy monitoring in patients with RA. Synovitis, for example, is an early manifestation of the disease that can reliably be detected by imaging procedures. Ultrasonography and MRI are able to detect synovitis, as well as increased synovial blood flow in affected joints, and nuclear medicine methods can also detect synovitis (imaging with nanocolloids, ${ }^{18}$ F-FDG PET, 3-phase bone scintigraphy). Several factors influence the use of imaging modalities in clinical practice. Ultrasonography, because it is sensitive, widely available, relatively inexpensive, and not associated with ionizing radiation, is gaining an increasingly prominent position as a screening modality for diagnosing synovitis. Furthermore, ultrasonography is convenient for patients and physicians. Therefore, it is a useful tool for the screening of suspected-RA patients for synovitis or inflammatory changes in other soft tissues such as tendons or tendon sheaths.
Also, ultrasonography may show bone erosions in small joints (finger and toe joints) with high sensitivity (27). However, ultrasonography is not suitable for visualizing erosions in larger joints because of its inability to penetrate bone. MRI, at this point in time, remains the gold standard for the detection of synovitis (28). It is also sensitive in the detection of erosions and, as compared with CT, offers the possibility to simultaneously evaluate erosions and synovitis. Plain radiography has been shown to be less sensitive in the detection of early lesions in RA $(28,29)$.

Planar bone scintigraphy has been used to image disease activity in patients who are receiving therapy, but because the method is clearly less sensitive than SPECT, early stages of the disease or mild abnormalities may be missed. Therefore, multipinhole SPECT of the hands has been used to identify patients with minimal changes in bone metabolism. Ostendorf et al. (30) demonstrated increased bone metabolism in finger joints by multipinhole SPECT. This technique proved to equal MRI in sensitivity and also detected increased bone metabolism in 2 patients in whom MRI had negative results (absence of bone edema), demonstrating that multipinhole SPECT may be even more sensitive than MRI in some cases.

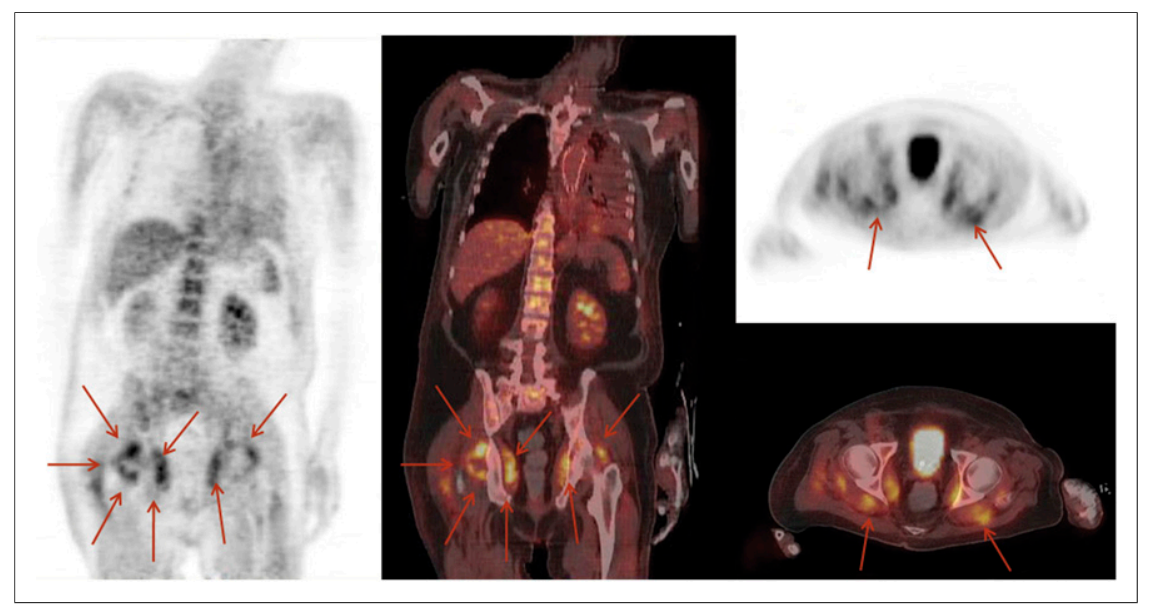

FIGURE 3. ${ }^{18} \mathrm{~F}-\mathrm{FDG}$ PET/CT scan of patient with FUO after placement of aortic stent for treatment of aneurysm of thoracic aorta. Stent infection was considered most likely cause of FUO, but ${ }^{18} \mathrm{~F}-\mathrm{FDG}$ PET showed bilaterally increased uptake in region of dorsal hip muscles related to abscess formation (arrows). This scan demonstrates value of whole-body PET in patients with FUO. 
High sensitivity and specificity have been reported for imaging of synovitis with ${ }^{99 \mathrm{~m}} \mathrm{Tc}$-nanocolloids, but in view of the broad availability of ultrasonography and MRI, whether this imaging technique will play a role remains more than questionable. As an alternative, PET may be used for imaging of synovial inflammation or arthritis. Besides ${ }^{18} \mathrm{~F}-\mathrm{FDG}$, tracers such as ${ }^{11} \mathrm{C}$-choline may be used for measurement of cell proliferation. This tracer detected synovitis with high accuracy (31). Again, the usefulness of PET, compared with MRI and ultrasonography, needs to be proven in RA imaging.

Currently, large systematic prospective studies on the efficient use of imaging modalities to assess the efficacy of treatment in early RA are lacking. For practical reasons, it seems as if many rheumatologists prefer MRI for early diagnosis of RA and use ultrasonography as a relatively inexpensive and easily available screening method after the history has been taken and physical examination and laboratory tests performed. Plain radiography can be used to exclude other bone diseases but is not sufficiently sensitive to detect early changes, detecting only (then largely irreversible) damage to joints at a later stage of the disease (28). Nuclear medicine techniques, especially bone scanning (including multipinhole SPECT of small joints) and PET, may significantly add to the early diagnosis of RA. Although PET and SPECT with either ${ }^{18} \mathrm{~F}-\mathrm{FDG}$ or bisphosphonates may play a role in identifying RA at an early stage $(28,30)$, repeated imaging to assess the efficacy of treatment may be required as often as every 6-12 wk over a long period (26), and the radiation exposure associated with both techniques will put ultrasonography and MRI at an advantage. As a future development in RA imaging, optical imaging may also become more important. The limited penetration of light does not play an important role in optical imaging of small joints (fingers and toes), and ligands specifically binding to inflamed synovial tissue may be able to detect RA at an early stage. This imaging technology is now limited mostly to preclinical use but may become an attractive modality in RA $(28,32)$.

In conclusion, the role of nuclear medicine imaging in the assessment of RA is currently not clear. The paradigmatic changes that have been taking place in the treatment of RA in the last 2 decades require highly sensitive imaging modalities that will also allow for repeated imaging. Although bone SPECT or ${ }^{18}$ F-FDG PET may play a role in the early diagnosis of RA, patient exposure to radiation hinders their use in repeated assessments. Large controlled, prospective clinical trials comparing different imaging modalities in RA diagnosis and therapy monitoring are lacking. Therefore, it is difficult to clearly position the use of SPECT or PET in RA. The actual use of nuclear medicine imaging techniques will depend more on local preferences than on true evidence from the literature. It seems likely that ultrasonography and MRI will become the most important techniques for RA imaging unless clinical trials demonstrate added value for PET or bone SPECT.

\section{Vasculitis}

The diagnosis of vasculitis is usually based on clinical symptoms, combined with laboratory tests. The American College of Rheumatology has defined criteria for the diagnosis of giant cell arteritis (GCA) and other types of vasculitis. Three of the following criteria have to be present to make the diagnosis of GCA probable: age greater than $50 \mathrm{y}$, new headache, abnormalities on palpation of temporal artery, elevated erythrocyte sedimentation rate, and histologic changes of the temporal artery. However, because vasculitis can often clinically present with unspecific symptoms, the diagnosis is not primarily considered. Therefore, in patients with unspecific symptoms caused by vasculitis, the underlying reason may not be detected by biopsy and histopathologic evaluation before vasculitis is identified as the potential cause. As an example, vasculitis may present as FUO (Fig. 4). In elderly patients, up to $17 \%$ of cases of FUO are caused by vasculitis (33). Furthermore, even if vasculitis is suspected, temporal artery biopsy is false-negative in up to $45 \%$ of patients (34). This exemplifies the potential value of imaging procedures in patients in whom the diagnosis remains unclear.

Ultrasonography has been shown to detect temporal arteritis with high sensitivity. If duplex ultrasonography is combined with Doppler ultrasonography, the diagnosis of arteritis is based mainly on 3 findings: edema visible as a hypoechoic circumferential wall thickening around the lumen, stenosis resulting in increased blood-flow velocity and turbulence, and occlusion resulting in loss of Doppler signal. If 1 or 2 of these signs are present, ultrasonography of arteritis will result in a sensitivity exceeding $85 \%$ and a specificity above $90 \%$ (35). Ultrasonography is suitable for imaging of the proximal arm and axillary arteries and can therefore play an important role in the detection of GCA or FUO caused by GCA (36). Ultrasonography can also play an important role in diagnosing small-vessel vasculitis (carotid, finger, and temporal arteries (37)). However, excellent results will be obtained only by experienced sonographers and may not be reached in general in clinical practice.

MRI also allows imaging of GCA with high sensitivity and specificity (81\% and 97\%, respectively) (38). Diagnostic criteria are thickening of the arterial walls and increased gadolinium contrast enhancement. However, high field strengths are required for imaging of vasculitis by MRI. One-tesla scanners seem to be inadequate for imaging of vasculitis, and a field strength of $1.5 \mathrm{~T}$ or, even better, $3 \mathrm{~T}$ is advised $(35,39)$. The reason is the trade-off between image resolution required for imaging of the walls of superficial cranial arteries and signal intensity.

${ }^{18} \mathrm{~F}-\mathrm{FDG}$ PET is a highly sensitive imaging technique for the detection of inflamed arterial walls. In contrast to ultrasonography, with ${ }^{18}$ F-FDG PET the thoracic aorta can also be visualized. Other large arteries can be visualized without the limitations associated with ultrasonography (gas in bowels, bones, locations deep within the body), allowing 


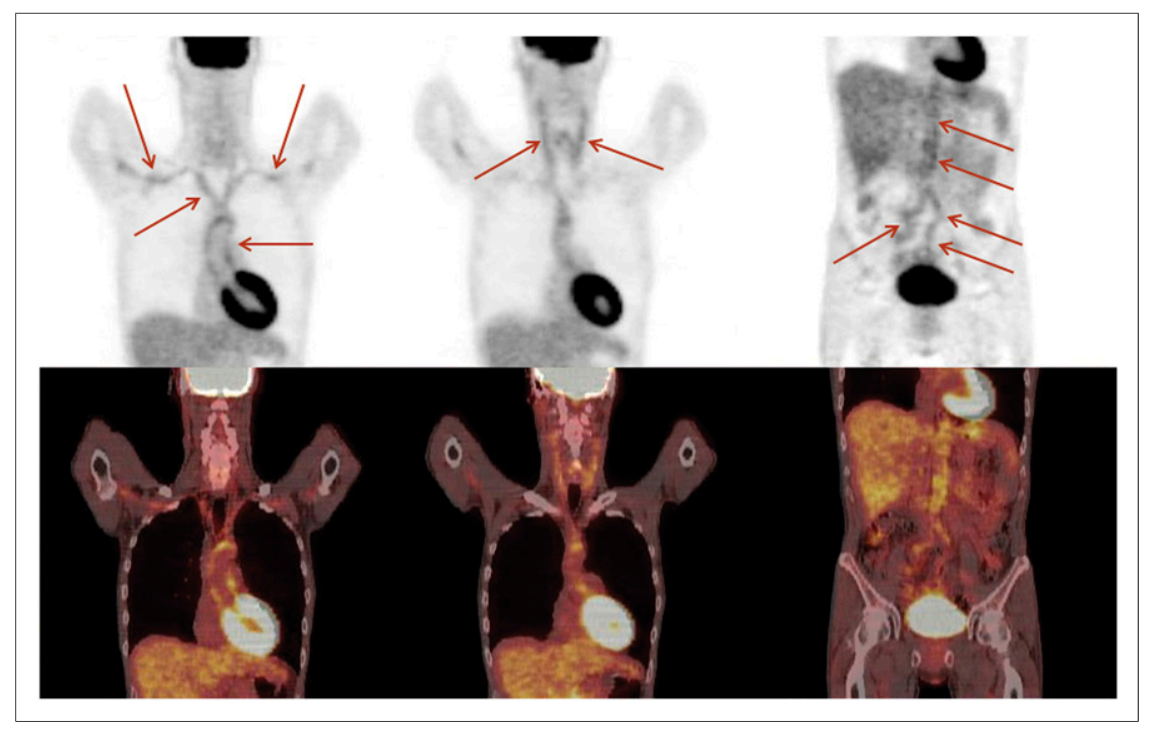

FIGURE 4. Patient with a history of FUO. Physical examination and ultrasonography of temporal artery were negative. Erythrocyte sedimentation and C-reactive protein were elevated; otherwise, laboratory parameters did not indicate vasculitis. ${ }^{18} \mathrm{~F}-\mathrm{FDG}$ PET scan shows increased uptake in thoracic aorta and subclavian arteries (left), carotid arteries (middle), and abdominal aorta together with iliac arteries (right) (arrows). Vasculitis was diagnosed. This example supports role of PET for detection of vasculitis as underlying cause of FUO. for simple assessment of the abdominal and pelvic arteries. ${ }^{18}$ F-FDG PET has been shown to have a high sensitivity of more than $80 \%$ in the detection of vasculitis of the large arteries (40). These results are in line with other data. Hautzel et al. have published a controlled study in which they not only demonstrated a high sensitivity for ${ }^{18} \mathrm{~F}-\mathrm{FDG}$ PET in the detection of vasculitis of the large arteries but also, on performing a receiver-operating-characteristic analysis comparing thoracic artery standardized uptake value with liver standardized uptake value, found an investigatorindependent sensitivity and specificity of $89 \%$ and $95 \%$ (41). When therapy of GCA was assessed with ${ }^{18} \mathrm{~F}-\mathrm{FDG}$ PET, the standardized uptake value decreased 3 mo after the onset of therapy but remained unchanged after 6 mo. This observation may be caused by vascular remodeling, and therefore PET seems to be less suitable for the evaluation of therapeutic effects (40). A study of biopsy-proven GCA patients demonstrated that vascular uptake of ${ }^{18} \mathrm{~F}$ FDG in the acute phase of disease correlated strongly with aortic diameter on follow-up (42). These findings suggest a role for ${ }^{18} \mathrm{~F}$-FDG PET not only in the primary diagnosis of disease but also as a prognostic tool for the development of aortic aneurysms. In patients with polymyalgia rheumatica, ${ }^{18}$ F-FDG uptake in the large arteries was detected in approximately $30 \%$ of patients, whereas increased uptake in the shoulders and hips was seen in $94 \%$ and $89 \%$, respectively. Of note, increased uptake was present in the processi spinosi of the cervical and lumbar spine in more than $50 \%$ of patients (43).

Patients with Takayasu arteritis (TA) are usually younger than patients with GCA at the onset of disease (40 y old or younger). Because the disease is rare, published studies have been performed on only a few patients. Several case reports are also available. In ultrasonography, the same changes are detected as in GCA, and the carotid and subclavian arteries are often involved $(44,45) .{ }^{18}$ F-FDG PET also offers good sensitivity and specificity exceeding $90 \%$ in patients with
TA, although the data have been obtained from small groups of patients $(46,47)$. It appears that slightly increased ${ }^{18} \mathrm{~F}$ FDG uptake may remain over the course of the disease, as is also the case for GCA (35). Contrast-enhanced MR arteriography seems to be a valuable imaging modality for detection and follow-up of TA. Although, in analogy to PET, the changes in the arterial walls tend to persist (rendering MR arteriography and MRI less suitable for assessment of disease activity (48)), MR arteriography as an anatomic imaging modality has the advantage of providing information about arterial stenosis as well as aneurysm formation, a common complication in TA (49).

In view of the available literature, ultrasonography appears appropriate as the primary imaging modality for the detection of typical signs of temporal arteritis, provided that the examination is performed by an experienced sonographer. Otherwise, the value of the method will be impaired. In patients in whom ultrasonography or temporal biopsy is negative or in whom GCA is only 1 possible differential diagnosis, ${ }^{18}$ F-FDG PET appears to be the logical second approach. Especially in FUO caused by vasculitis, PET plays a pivotal role in the diagnosis (Fig. 4) (50). The advantages of PET are the high sensitivity and the ability to screen all large arteries in a single step (standardized whole-body scanning). Furthermore, uptake of ${ }^{18}$ F-FDG seems to correlate with later formation of aneurysms, thus rendering ${ }^{18} \mathrm{~F}-\mathrm{FDG}$ uptake a prognostic marker. However, PET has a low sensitivity only in the detection of small (and medium)-vessel disease, and imaging such disease is the strength of MRI, provided that imaging of a given anatomic region is performed. Thus, MRI and MR arteriography are highly sensitive for the detection of changes in the superficial cranial arteries. Furthermore, MRI and MR arteriography allow for the detection of aneurysm formation and are thus especially useful in the diagnosis and follow-up of TA. Blockmans et al. demonstrated a decrease of ${ }^{18} \mathrm{~F}$-FDG uptake in diseased vessels 3 mo after the initiation of therapy (35). However, 
the uptake did not further decrease 6 mo after therapy. This persisting ${ }^{18} \mathrm{~F}$-FDG uptake seems to be caused by vessel wall remodeling. Both PET and MRI appear to show this vessel wall remodeling in GCA and TA; after assessment of the initial therapeutic response by both modalities, changes in the vessel walls will persist. Therefore, more data are required to elucidate the potential of PET and MRI in monitoring the response of vasculitis to therapy later than 6 mo (35). When PET scans are evaluated for the presence of vasculitis, these factors need to be kept in mind. If the patient is receiving immunosuppressive therapy, uptake into diseased vessels will be decreased but not absent. Slightly increased vessel uptake may reflect vascular remodeling and may be a sign of vasculitis in patients receiving immunosuppression.

${ }^{18}$ F-FDG PET may be a helpful tool in diagnosing polymyalgia rheumatica, because the large arteries are less frequently involved in this disease than in GCA or TA (35). Increased uptake in the shoulders or hips together with increased uptake in the cervical and lumbar processi spinosi may point toward polymyalgia rheumatica, and involvement of the large vessels can be found in around $30 \%$ of patients (43).

\section{Sarcoidosis}

Most frequently, sarcoidosis affects the lungs and the associated hilar and mediastinal lymph nodes. Typically, noncaseating granulomas are found. However, because sarcoidosis can affect multiple organ systems, the clinical presentation can vary considerably. Unspecific constitutional symptoms can be found frequently; more specifically, dyspnea and coughing are associated with lung involvement. On chest radiographs, mediastinal and bilateral hilar lymphadenopathy can be found; less frequently found are parenchymal opacities and, in advanced stages of disease, pulmonary fibrosis, as is reflected in a staging system ranging from 0 (no abnormality) to 4 (pulmonary fibrosis) based on chest radiography findings (51). Peripheral lymphadenopathy is also frequently found in up to one third of patients (cervical, axillary, inguinal), whereas skin lesions affect about one quarter of patients (51).

Chest radiographs still play a role in the detection of sarcoidosis in patients presenting with dyspnea and coughing. CT has achieved a central role in detecting lymphadenopathy and changes in lung parenchyma on high-resolution scans. Nuclear imaging techniques used for the assessment of sarcoidosis include ${ }^{67} \mathrm{Ga}$ imaging and ${ }^{18} \mathrm{~F}-\mathrm{FDG}$ PET. These allow disease activity to be determined and treatment response monitored $(6,52)$. In particular, both techniques have been reported to detect sites of disease previously unknown, as well as unsuspected metabolically active disease. This aspect is of particular importance, as PET detects multiorgan involvement in a considerable number of patients (53). Furthermore, PET is superior to ${ }^{67} \mathrm{Ga}$ imaging and is able to detect sites of inflammation unrecognized by ${ }^{67} \mathrm{Ga}$ imaging $(54,55)$.

In the primary diagnosis of sarcoidosis, high ${ }^{18} \mathrm{~F}-\mathrm{FDG}$ uptake may be present in lymph nodes and in other involved organs. This uptake may mimic malignant disease such as lymphoma (Fig. 5) (6). The same problem occurs in CT of patients with sarcoidosis, as enlarged abdominal lymph nodes may be mistaken for lymphoma, metastatic disease, or Mycobacterium infection (56). Definitive diagnosis often requires biopsy to prove sarcoidosis and to exclude other (malignant) diseases. In response to treatment, uptake of ${ }^{67} \mathrm{Ga}$ and of ${ }^{18} \mathrm{~F}$-FDG decreases, allowing for assessment of treatment effects and for early adaptation of therapy if no response can be measured $(53,57)$. Also, patients with pulmonary fibrosis who may not profit from corticosteroid therapy can be identified (53).

Therefore, in the assessment of sarcoidosis, PET with ${ }^{18} \mathrm{~F}-\mathrm{FDG}$ allows disease activity and treatment response to be determined. ${ }^{18} \mathrm{~F}$-FDG PET is superior to ${ }^{67} \mathrm{Ga}$ imaging. Traditionally, chest radiography and CT have played an important role in the diagnosis of sarcoidosis. In view of the advantages of combined PET/CT, one should consider replacing several CT scans by combined whole-body PET/ CT. For determination of changes in the lung parenchyma, high-resolution CT is most suitable.

\section{IBD}

Crohn's disease and ulcerative colitis are the 2 main subtypes of IBD. Although ulcerative colitis affects solely the colon, Crohn's disease can affect any part of the gastrointestinal tract, with involvement of the terminal ileum in $90 \%$. Consequently, ileocolonoscopy has gained wide acceptance as the reference standard for diagnosing colonic or ileal involvement. For small-bowel disease, barium examinations using an enteroclysis technique are still considered the standard of reference (58). Furthermore, disease activity is measured clinically by clinical activity scores. This diagnostic approach has several disadvantages: the clinical scoring system is indirect and endoscopy or barium enteroclysis are both invasive techniques. Barium may be contraindicated

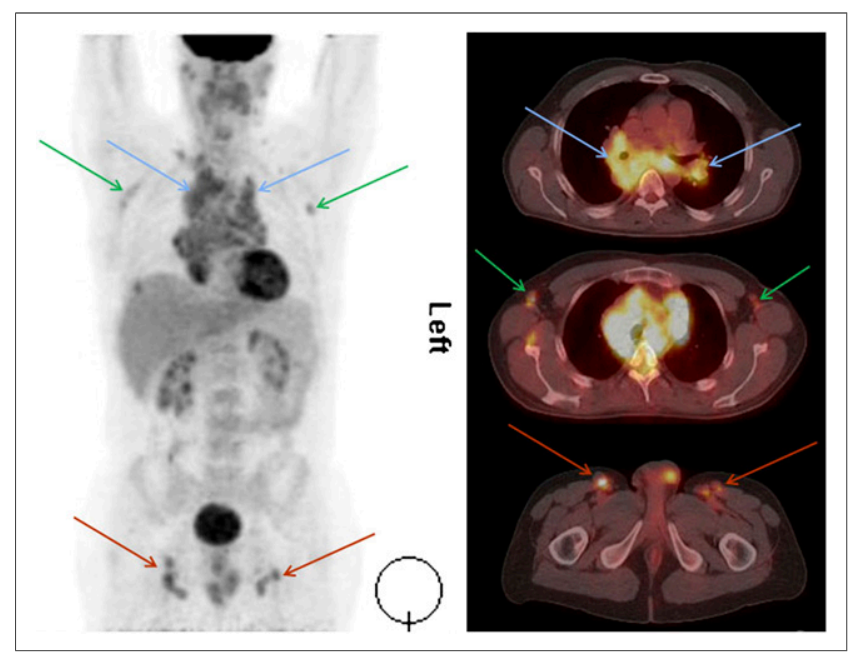

FIGURE 5. ${ }^{18} \mathrm{~F}-\mathrm{FDG} \mathrm{PET} / \mathrm{CT}$ scan of patient with sarcoidosis. Maximal-intensity projection on left side gives overview of sites of disease, including cervical area, axillae (green arrows), mediastinum (blue arrows), and inguinal lymph nodes (red arrows). 
in cases of severe inflammation because of the risk of perforation and subsequent peritonitis. In acute ulcerative colitis with severe inflammation, colonoscopy is associated with an increased risk of perforation. Therefore, noninvasive imaging procedures are warranted to adequately diagnose and follow patients with IBD (59).

WBC imaging (preferably with ${ }^{99 \mathrm{~m}} \mathrm{Tc}$ instead of ${ }^{111} \mathrm{In}$ because of better image quality) has extensively been used for diagnosing IBD. This imaging technique has also shown high sensitivity and specificity between $80 \%$ and $90 \%$ in adults and children for the correct detection of the involved bowel segment (60-63). WBC scanning may also help to diagnose therapy resistance within days after the onset of therapy (64). However, as a consequence of the rather complicated and time-consuming labeling procedure of WBCs and the duration of the procedure, WBC scanning is relatively inconvenient for the patient and requires a dedicated laboratory and personnel for handling of blood products.

Horsthuis et al. have performed a metaanalysis of prospective studies on imaging of IBD by ultrasonography, MRI, CT, and WBC scintigraphy (62). They demonstrated that CT is less sensitive than ultrasonography, MRI, and WBC scanning and advised that CT not be performed in the diagnostic work-up of IBD. Ultrasonography, MRI, and WBC scanning were equivalent in IBD imaging, with sensitivities ranging from $84 \%$ to $93 \%$ and specificities ranging from $84 \%$ to $95 \%$. Ultrasonography is very much operatordependent, and usually not all bowel segments can be visualized; therefore, the use of this imaging modality may depend on locally available expertise. The authors advised the primary use of ultrasonography and MRI (using an enteroclysis technique rather than oral administration of contrast medium) for IBD imaging. This advice was based mainly on the absence of radiation exposure of ultrasonography and MRI. Moreover, PET has not been evaluated in this metaanalysis because of the limited number of published studies (62).

${ }^{18}$ F-FDG PET has been evaluated in children and adults with IBD. In a study of 65 children, PET had a good sensitivity of $80 \%$ (compared with colonoscopy), and a negative PET scan excluded the presence of inflammation in children with recurrent abdominal pain (65). In another study of children, the results of PET were compared with histology, and PET reached a sensitivity of $98 \%$ and a specificity of $68 \%$ (in comparison to endoscopy [90\% and $75 \%$, respectively] and ultrasonography [56\% and 92\%, respectively]). For small-bowel disease, PET was even more reliable (65). In a study of adults with IBD, hydro-MRI was clearly inferior to PET, with sensitivities of $40.9 \%$ and $85.4 \%$, respectively (66). More studies have demonstrated a good sensitivity for PET in IBD, with a high correlation with disease activity, laboratory parameters, endoscopy, and other parameters (67-70).

The role of video capsule endoscopy in the evaluation of IBD is not completely clear. Although promising results have been obtained, questions remain. For example, the correlation between mucosal healing diagnosed by video capsule endoscopy and follow-up is not clear. Before video capsule endoscopy can be performed, one should be certain that there are no strictures, which would lead to retention of the capsule and, possibly, bowel obstruction (71). Thus, the technique is not without risk of complications. Indeed, retention of the capsule was observed in more than $10 \%$ of the patients with Crohn's disease (72). However, video capsule endoscopy is a highly promising technique whose exact role in IBD will have to be determined.

For imaging of IBD, ${ }^{18}$ F-FDG PET seems to be equivalent in sensitivity to WBC, although supporting data are limited so far. For WBC imaging, SPECT/CT should be performed for the correct localization of involved bowel segments. In conclusion, ultrasonography is a valuable tool for imaging of IBD but, because of its limitations (operator dependence, bowel segments not completely visible), should be followed by MRI, WBC imaging, or PET (Fig. 6). Insufficient data are available to conclude whether MRI or nuclear medicine techniques are preferable for imaging of IBD. Prospective controlled trials comparing PET or WBC imaging and MRI, and their influence on therapy in IBD, are warranted.

\section{IMAGING IN FUO}

FUO was originally defined in 1961 as a temperature of at least $38.3^{\circ} \mathrm{C}\left(101^{\circ} \mathrm{F}\right)$ on several occasions, with the underlying cause being uncertain after $1 \mathrm{wk}$ of hospitalization. The potential causes of FUO include a broad spectrum of diseases, namely infectious diseases (such as abscess or endocarditis), autoimmune disorders (such as vasculitis or rheumatic disease), and malignant tumors. In the past 4 decades, new imaging and other diagnostic techniques have been developed, allowing earlier diagnosis in many patients with fever. For example, ultrasonography, CT, or MRI together with laboratory tests have facilitated the diagnosis of malignant diseases; echocardiography allows for a reliable diagnosis of endocarditis. Immunodeficient patients are not included in the FUO definition because they need an entirely different approach, which does not fall into the scope of this article. Recently, the criteria for definition of FUO have been modified to a list of investigations that need to be performed to identify the origin of the fever. If the diagnosis is still uncertain after these tests, the patient is classified as having FUO (73).

In the clinical work-up of a patient with FUO, it is essential to first identify clues about the diagnosis by taking a thorough history and performing a physical examination followed by laboratory tests, chest radiography, and abdominal ultrasonography. If a hypothesis for the cause of FUO can be established on the basis of these findings, a further focused diagnostic work-up can be performed to prove or refute the hypothesis (73).

Imaging techniques may help to identify the cause of FUO. Anatomic imaging modalities require anatomic changes in tissues secondary to inflammation before detection is 


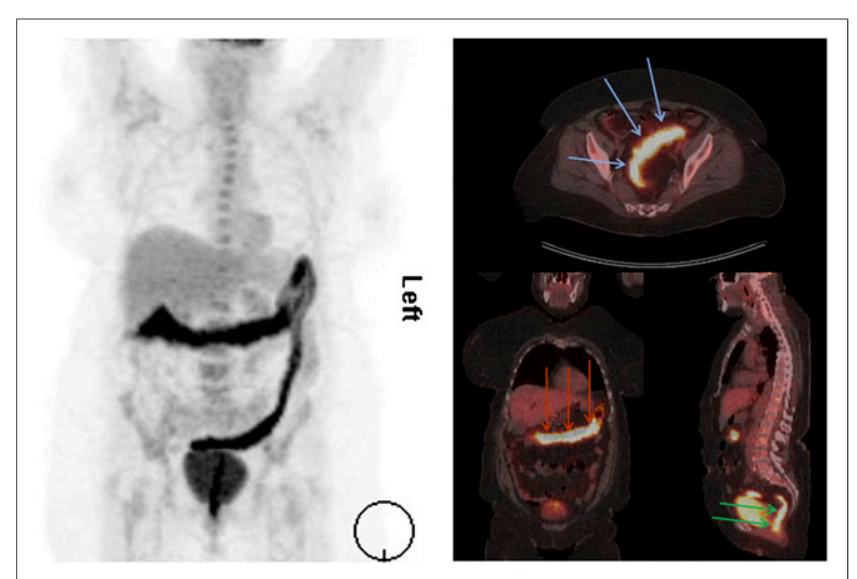

FIGURE 6. ${ }^{18} \mathrm{~F}-\mathrm{FDG}$ PET/CT scan of patient with ulcerative colitis. Maximal-intensity projection on left side gives overview of extent of disease, which includes transverse colon (red arrows on coronal slice), sigmoid (blue arrows on transversal slice), and rectum (green arrows on sagittal slice).

possible and therefore in many cases are not able to detect early stages of disease. In addition, changes caused by cured disease or surgery (scars, fibrosis, and other causes) may result in false-positive interpretation of the images. Furthermore, anatomic imaging modalities routinely include only a limited area of the body and do not provide information about other areas-a major disadvantage if the potential cause of the fever is not clear. Therefore, nuclear medicine imaging techniques play an important role in the diagnosis of FUO because they are performed as wholebody imaging examinations and the radiotracers used are able to detect inflammatory processes in the body at an early stage, possibly before anatomic changes appear.

Scintigraphic detection of inflammatory foci using ${ }^{67} \mathrm{Ga}$ has been widely performed, and although this method has proven efficiency in the detection of inflammation, it is associated with several disadvantages. Because of the high $\gamma$-energy, image quality is impaired by lower resolution resulting from the use of high-energy collimators and other factors. Furthermore, the radiopharmaceutical has to clear from the background to achieve acceptable target-to-background ratios (especially, bowel uptake resulting from clearance via the liver disturbs imaging), often requiring late images to be obtained (48- or 72-h imaging). Therefore, ${ }^{67} \mathrm{Ga}$ imaging has several disadvantages for patients: it is timeconsuming, must be repeated, and has a relatively high radiation burden. In a study of 58 patients with FUO, Meller et al. showed that ${ }^{67} \mathrm{Ga}$ scintigraphy had a diagnostic yield nearly equivalent to that of ${ }^{18}$ F-FDG PET (74). However, in that study, ${ }^{18} \mathrm{~F}-\mathrm{FDG}$ scanning was performed with a coincidence camera instead of a dedicated full-ring PET scanner, which is superior for PET. In view of the disadvantages of ${ }^{67} \mathrm{Ga},{ }^{18} \mathrm{~F}$-FDG PET is the procedure of choice for this indication. The advantage of ${ }^{18} \mathrm{~F}-\mathrm{FDG}$ is the high rate of background clearance achieved via renal elimination while the tracer is trapped in cells with high glucose consumption.
Therefore, tissues with increased glucose metabolism can be identified with high sensitivity. Because both inflammatory cells and tumor cells have a high glucose demand, ${ }^{18} \mathrm{~F}-\mathrm{FDG}$ PET is highly suitable for the detection of both inflammation and malignancy. ${ }^{18}$ F-FDG PET has high sensitivity for most malignant tumors. In addition, the therapeutic and prognostic consequences of a delay in diagnosing a malignancy, responsible for FUO in up to $25 \%$ of cases, are clinically important. Therefore, high accuracy in diagnosing malignant disease should be an important operational characteristic for recommended nuclear medicine techniques in FUO patients. ${ }^{18}$ F-FDG PET is also able to detect bacterial inflammation and most types of autoimmune inflammation (Fig. 4). Therefore, all important underlying causes of FUO (infection, autoimmune disease, malignancy) can usually be detected with high sensitivity by a single imaging technique.

As another alternative, labeled leukocytes may be used to identify infectious foci. However, because leukocytes accumulate mainly in foci of bacterial infection and the prevalence of FUO caused by bacterial infections is relatively low $(15 \%-40 \%)$, the evaluation of patients with other underlying diseases (malignancies, autoimmune diseases) will mostly be false-negative (73), and the clinical value of labeled leukocytes for diagnosing the cause of FUO is therefore limited.

A potential drawback of ${ }^{18} \mathrm{~F}-\mathrm{FDG}$ is physiologic accumulation in the brain, heart, bowel, and urinary tract due to excretion of the tracer via urine. In addition, in patients with fever, uptake into the bone marrow may be increased. PET is not suitable for diagnosing endocarditis as a cause of FUO. In patients with suspected endocarditis, echocardiography is the imaging modality of choice. Other diseases, such as IBD or pyelonephritis, are usually associated with typical clinical symptoms; neither condition is a typical cause of FUO, and they are therefore less relevant for PET in FUO. The diffusely increased uptake of ${ }^{18}$ F-FDG in the bone marrow usually shows a typical pattern, and the diagnostic accuracy of PET would be impaired only if a small focus of equivocal uptake in the bone marrow were present.

Because of its high sensitivity, favorable imaging characteristics (good spatial resolution, no delayed imaging required), whole-body imaging, and high patient convenience, ${ }^{18}$ F-FDG PET currently is the most powerful imaging modality in the diagnosis of FUO, especially when combined with CT in integrated PET/CT scanners allowing exact anatomic localization of ${ }^{18} \mathrm{~F}-\mathrm{FDG}$-avid foci. Therefore, with the advent of combined PET/CT, PET has gained considerably in specificity.

In FUO, it is of the utmost importance to identify a focus as the underlying cause of the disease. Therefore, high sensitivity combined with exact localization of foci-both characteristics inherent to PET/CT-plays a pivotal role in the diagnosis of FUO.

In several studies, ${ }^{18}$ F-FDG PET has shown value in the diagnosis of FUO. Although it is difficult to compare those studies because of the differences in inclusion criteria and 
the different time points in the diagnostic work-up at which PET was performed, ${ }^{18} \mathrm{~F}$-FDG PET showed an overall helpfulness, corrected for study population, of approximately $35 \%-40 \%$, which is high compared with radiologic techniques and ${ }^{67} \mathrm{Ga}$-citrate scintigraphy $(73,75-79)$. In 1 prospective study, all patients had undergone a clearly defined standardized diagnostic evaluation before PET was performed. In addition, that study compared PET/CT with CT of the abdomen and chest (76). The study clearly showed that ${ }^{18} \mathrm{~F}-\mathrm{FDG}$ PET is superior to CT in patients with FUO. So far, in the absence of systematic evaluation of CT and MRI in patients with FUO, this has been the only study systematically comparing PET and CT. Furthermore, PET did not contribute to the diagnosis of FUO when $\mathrm{C}$-reactive protein and erythrocyte sedimentation rate were normal. On the basis of these results and the favorable characteristics of ${ }^{18} \mathrm{~F}-\mathrm{FDG}$ PET, conventional scintigraphic techniques should be replaced by ${ }^{18} \mathrm{~F}$-FDG PET in the investigation of patients with FUO in institutions where this technique is available. Also, in comparison to CT, ${ }^{18} \mathrm{~F}-\mathrm{FDG}$ PET performs excellently. Therefore, ${ }^{18}$ F-FDG PET should be performed early if imaging techniques are used in the diagnostic work-up of patients with FUO.

\section{CONCLUSION}

Nuclear medicine imaging techniques play an important role in the assessment of inflammatory diseases. However, there is strong competition with other imaging modalities, especially ultrasonography, MRI, and CT. Here, we summarize our advice for the use of the different imaging modalities based on the current literature:

- In the diagnosis of osteomyelitis and RA, MRI has gained wide acceptance together with ultrasonography. However, with the advent of new SPECT reconstruction algorithms and multipinhole SPECT acquisition, nuclear medicine imaging techniques may still have a role in the assessment of RA, and clinical studies are required to define the future role of SPECT in RA. PET is highly suitable for imaging of spondylitis and spondylodiscitis and may also play a role in the assessment of osteomyelitis.

- In FUO, PET with ${ }^{18} \mathrm{~F}-\mathrm{FDG}$ is able to play a key role in the assessment of patients in whom clinical and laboratory findings together with ultrasonography have not revealed a diagnosis. As long as comparative studies between MRI, PET, and CT are largely missing, the available data suggest preferring PET. Furthermore, vasculitis may cause FUO in a considerable number of patients, further supporting the central role of ${ }^{18} \mathrm{~F}$ FDG PET in FUO.

- In IBD, PET may also play an important role in the future. WBC has performed convincingly in comparison to MRI and CT.

- In vasculitis, PET seems to be of crucial importance if the diagnosis cannot be made on the basis of temporal artery biopsy and ultrasonography. This is especially true for GCA and polymyalgia rheumatica, whereas in TA, MRI or MR arteriography will enable a better detection of aneurysms.

- ${ }^{18} \mathrm{~F}-\mathrm{FDG}$ PET is a highly sensitive imaging modality in diagnosing sarcoidosis and also allows for assessment of treatment response.

- ${ }^{18} \mathrm{~F}-\mathrm{FDG}$ PET/CT is the procedure of choice in the diagnosis of vascular graft infection; simultaneous CT is mandatory to avoid false-positive results. PET scans needs to be assessed according to the mentioned criteria to avoid false-positive results. Patients with nondiagnostic PET scans should undergo WBC scanning.

- PET can detect metastatic infectious foci with high sensitivity, even if other imaging procedures show negative results.

- ${ }^{67} \mathrm{Ga}$ imaging can largely be replaced by ${ }^{18} \mathrm{~F}-\mathrm{FDG}$ PET, as ${ }^{67} \mathrm{Ga}$ offers no advantages for imaging of inflammatory diseases.

- WBC scanning may still play a role (IBD, vascular graft infection) and have some advantages over PET in certain categories of patients. However, WBC scanning requires handling of blood products, and the labeling is time-consuming and inconvenient for the patient, who has to wait for labeling and imaging. Therefore, for practical reasons, WBC scanning is becoming less popular. Another disadvantage is that it may need to be combined with other imaging techniques to achieve high sensitivity and specificity.

\section{REFERENCES}

1. Palestro CJ, Kipper SL, Weiland FL, et al. Osteomyelitis: diagnosis with ${ }^{99 m} \mathrm{Tc}-$ labeled antigranulocyte antibodies compared with diagnosis with ${ }^{111}$ In-labeled leukocytes-initial experience. Radiology. 2002;223:758-764.

2. Hakim SG, Bruecker CW, Jacobsen H, et al. The value of FDG-PET and bone scintigraphy with SPECT in the primary diagnosis and follow-up of patients with chronic osteomyelitis of the mandible. Int J Oral Maxillofac Surg. 2006;35: 809-816.

3. Horger M, Eschmann SM, Pfannenberg C, et al. Added value of SPECT/CT in patients suspected of having bone infection: preliminary results. Arch Orthop Trauma Surg. 2007;127:211-221.

4. van der Bruggen W, Bleeker-Rovers CP, Boerman OC, Gotthardt M, Oyen WJ. PET and SPECT in osteomyelitis and prosthetic joint infections: a systematic review. Semin Nucl Med. 2010;40:3-15.

5. Palestro CJ, Love C, Tronco GG, et al. Combined labeled leukocyte and technetium $99 \mathrm{~m}$ sulfur colloid bone marrow imaging for diagnosing musculoskeletal infection. Radiographics. 2006;26:859-870.

6. Basu S, Zhuang H, Torigian DA, Rosenbaum J, Chen W, Alavi A. Functional imaging of inflammatory diseases using nuclear medicine techniques. Semin Nucl Med. 2009;39:124-145.

7. Kälicke T, Schmitz A, Risse JH, et al. Fluorine-18 fluorodeoxyglucose PET in infectious bone diseases: results of histologically confirmed cases. Eur J Nucl Med. 2000;27:524-528.

8. Meller J, Koster G, Liersch T, et al. Chronic bacterial osteomyelitis: prospective comparison of ${ }^{18} \mathrm{~F}$-FDG imaging with a dual-head coincidence camera and ${ }^{111} \mathrm{In}$ labelled autologous leucocyte scintigraphy. Eur J Nucl Med Mol Imaging. 2002; 29:53-60.

9. Gratz S, Dorner J, Fischer U, et al. ${ }^{18}$ F-FDG hybrid PET in patients with suspected spondylitis. Eur J Nucl Med Mol Imaging. 2002;29:516-524.

10. de Winter F, van de Wiele C, Vogelaers D, et al. Fluorine-18 fluorodeoxyglucoseposition emission tomography: a highly accurate imaging modality for the diagnosis of chronic musculoskeletal infections. J Bone Joint Surg Am. 2001;83-A:651-660.

11. de Winter F, Gemmel F, van de Wiele C, Poffijn B, Uyttendaele D, Dierckx R. 18-fluorine fluorodeoxyglucose positron emission tomography for the diagnosis 
of infection in the postoperative spine. Spine (Phila Pa 1976). 2003;28:13141319.

12. Palestro CJ, Love C, Miller TT. Imaging of musculoskeletal infection. Best Pract Res Clin Rheumatol. 2006;20:1197-1218.

13. Schwegler B, Stumpe KD, Weishaupt D, et al. Unsuspected osteomyelitis is frequent in persistent diabetic foot ulcer and better diagnosed by MRI than by ${ }^{18}$ F-FDG or ${ }^{99 m}$ Tc-MOAB. J Intern Med. 2008;263:99-106.

14. Nawaz A, Torigian DA, Siegelman ES, Basu S, Chryssikos T, Alavi A. Diagnostic performance of FDG-PET, MRI, and plain film radiography (PFR) for the diagnosis of osteomyelitis in the diabetic foot. Mol Imaging Biol. 2009;12: $335-342$.

15. Hirsch W, Krohmer S, Kluge R, et al. Preliminary results in whole-body MRI in children: a prospective study. Pediatr Radiol. 2005;35:S89.

16. Darge K, Jaramillo D, Siegel MJ. Whole-body MRI in children: current status and future applications. Eur J Radiol. 2008;68:289-298.

17. Van Hoorn R, Vriens D, Postema JP, et al. Evaluation of the of advanced ReSPECT image reconstruction software in a phantom model and in parathyroid scanning [abstract]. Nuklearmedizin. 2010;49:A9.

18. Chang JK, Calligaro KD, Ryan S, Runyan D, Dougherty MJ, Stern JJ. Risk factors associated with infection of lower extremity revascularization: analysis of 365 procedures performed at a teaching hospital. Ann Vasc Surg. 2003;17: 91-96.

19. Keidar Z, Nitecki S. FDG-PET for the detection of infected vascular grafts. $Q J$ Nucl Med Mol Imaging. 2009;53:35-50.

20. Fukuchi K, Ishida Y, Higashi M, et al. Detection of aortic graft infection by fluorodeoxyglucose positron emission tomography: comparison with computed tomography findings. J Vasc Surg. 2005;42:919-925.

21. Keidar Z, Engel A, Hoffmann A, Israel O, Nitecki S. Prosthetic vascular graft infection: the role of ${ }^{18}$ F-FDG-PET/CT. J Nucl Med. 2007;48:1230-1236.

22. Spacek M, Belohlavek O, Votrubova J, Sebesta P, Stadler P. Diagnostics of "nonacute" vascular prosthesis infection using ${ }^{18} \mathrm{~F}-\mathrm{FDG}$ PET/CT: our experience with 96 prostheses. Eur J Nucl Med Mol Imaging. 2009;36:850-858.

23. Gardet E, Addas R, Monteil J, Guyader A. Comparison of detection of F-18 fluorodeoxyglucose positron emission tomography and ${ }^{99 \mathrm{~m}} \mathrm{Tc}$-hexamethylpropylene amine oxime labelled leukocyte scintigraphy for an aortic graft infection. Interact Cardiovasc Thorac Surg. 2010;10:142-143.

24. Bleeker-Rovers CP, Vos FJ, Corstens FHM, Oyen WJG. Imaging of infectious diseases using ${ }^{18}$ F-fluorodeoxyglucose PET. Q J Nucl Med Mol Imaging. 2008; 52:17-29.

25. Vos FJ, Bleeker-Rovers CP, Sturm P, et al. ${ }^{18}$ F-FDG PET/CT for detection of metastatic infection in Gram-positive bacteremia. J Nucl Med. 2010;51: 1234-1240

26. Smolen JS, Aletaha D, Bijlsma JWJ, et al. Treating rheumatoid arthritis to target: recommendations of an international task force. Ann Rheum Dis. 2010;69: 631-637.

27. Szkudlarek M, Narvestad E, Klarlund M, et al. Ultrasonography of the metatarsophalangeal joints in rheumatoid arthritis, compared with magnetic resonance imaging, conventional radiography and clinical examination. Arthritis Rheum. 2004;50:2103-2112.

28. McQueen FM, Østergaard M. Established rheumatoid arthritis: new imaging modalities. Best Pract Res Clin Rheumatol. 2007;21:841-856.

29. Hoving JL, Buchbinder R, Hall S, et al. A comparison of magnetic resonance imaging, sonography, and radiography of the hand in patients with early rheumatoid arthritis. J Rheumatol. 2004;31:663-675.

30. Ostendorf B, Mattes-György K, Reichelt DC, et al. Early detection of bony alterations in rheumatoid and erosive arthritis of finger joints with high-resolution single photon emission computed tomography, and differentiation between them. Skeletal Radiol. 2010;39:55-61.

31. Roivainen A, Parkkola R Yli-Kerttula T, et al. Use of positron emission tomography with methyl-11choline and 2-18fluoro-2-deoxy-D-glucose in comparison with magnetic resonance imaging for the assessment of inflammatory proliferation of synovium. Arthritis Rheum. 2003;48:3077-3084.

32. Wunder A, Schellenberger E, Mahmood U, et al. Methotrexate induced accumulation of fluorescent annexin $\mathrm{V}$ in collagen-induced arthritis. Mol Imaging. 2005;4:1-6.

33. Knockaert DC, Vanneste LJ, Bobbaers HJ. Fever of unknown origin in elderly patients. J Am Geriatr Soc. 1993;41:1187-1192.

34. Chong EW, Robertson AJ. Is temporal artery biopsy a worthwhile procedure? ANZ J Surg. 2005;75:388-391.

35. Blockmans D, Bley T, Schmidt W. Imaging for large vessel vasculitis. Curr Opin Rheumatol. 2009;21:19-28.

36. Schmidt WA, Seifert A, Gromnica-Ihle E, et al. Ultrasound of proximal upper extremity arteries to increase the diagnostic yield in large-vessel giant cell arteritis. Rheumatology. 2008;47:96-101.
37. Schmidt WA, Krause A, Schicke B, Wernicke D. Color Doppler ultrasonography of hand and finger arteries to differentiate primary from secondary forms of Raynaud's phenomenon. J Rheumatol. 2008;35:1591-1598.

38. Blockmans D, De Ceuninck L, Vanderschueren S, et al. Repetitive 18-fluorodeoxyglucose positron emission tomography in isolated polymyalgia rheumatica: a prospective study in 35 patients. Rheumatology. 2007;46:672-677.

39. Pipitone N, Versari A, Salvarani C. Role of imaging studies in the diagnosis and follow-up of large-vessel vasculitis: an update. Rheumatology. 2008;47:403-408.

40. Blockmans D, De Ceuninck L, Vanderschueren S, et al. Repetitive ${ }^{18} \mathrm{~F}$ fluorodeoxyglucose positron emission tomography in giant cell arteritis: a prospective study of 35 patients. Arthritis Rheum. 2006;55:131-137.

41. Hautzel H, Sander O, Heinzel A, et al. Assessment of large-vessel involvement in giant cell arteritis with ${ }^{18} \mathrm{~F}$-FDG PET: introducing an ROC-analysis-based cutoff ratio. J Nucl Med. 2008;49:1107-1113.

42. Bley TA, Uhl M, Carew J, et al. Diagnostic value of high-resolution MR imaging in giant cell arteritis. Am J Neuroradiol. 2007;28:1722-1727.

43. Blockmans D, Coudyzer W, Vanderschueren S, et al. Relationship between fluorodeoxyglucose uptake in the large vessels and late aortic diameter in giant cell arteritis. Rheumatology. 2008;47:1179-1184.

44. Andrews J, Mason JC. Takayasu's arteritis: recent advances in imaging offer promise. Rheumatology. 2007;46:6-15.

45. Schmidt WA, Seipelt E, Krause A, Wernicke D. Carotidynia in Takayasu arteritis. J Rheumatol. 2007;34:231-232.

46. Webb M, Chambers A, Al-Nahhas A, et al. The role of ${ }^{18} \mathrm{~F}-\mathrm{FDG}$ PET in characterising disease activity in Takayasu arteritis. Eur J Nucl Med Mol Imaging. 2004;31:627-634.

47. Andrews J, Al-Nahhas A, Pennell DJ, et al. Non-invasive imaging in the diagnosis and management of Takayasu's arteritis. Ann Rheum Dis. 2004;63:995-1000.

48. Tso E, Flamm SD, White RD, et al. Takayasu's arteritis: utility and limitations of magnetic resonance imaging in diagnosis and treatment. Arthritis Rheum. 2002; 46:1634-1642.

49. Gornik HL, Creager MA. Aortitis. Circulation. 2008;117:3039-3051.

50. Meller J, Sahlmann CO, Gürocak O, Liersch T, Meller B. FDG-PET in patients with fever of unknown origin: the importance of diagnosing large vessel vasculitis. Q J Nucl Med Mol Imaging. 2009;53:51-63.

51. Statement on sarcoidosis. Joint Statement of the American Thoracic Society (ATS), the European Respiratory Society (ERS), and the World Association of Sarcoidosis and Other Granulomatous Disorders (WASOG) adopted by the ATS Board of Directors and by the ERS Executive Committee, February 1999. Am J Respir Crit Care Med. 1999;160:736-755.

52. Aide $\mathrm{N}$, Benayoun $\mathrm{M}$, Kerrou $\mathrm{K}$, et al. Impact of $\left[{ }^{18} \mathrm{~F}\right]$-fluorodeoxyglucose $\left(\left[{ }^{18} \mathrm{~F}\right]-\mathrm{FDG}\right)$ imaging in sarcoidosis: unsuspected neurosarcoidosis discovered by $\left[{ }^{18} \mathrm{~F}\right]$-FDG PET and early metabolic response to corticosteroid therapy. $\mathrm{Br}$ J Radiol. 2007;80:e67-e71.

53. Teirstein AS, Machac J, Almeida O, et al. Results of 188 whole-body fluorodeoxyglucose positron emission tomography scans in 137 patients with sarcoidosis. Chest. 2007;132:1949-1953.

54. Xiu Y, Yu JQ, Cheng E, et al. Sarcoidosis demonstrated by FDG PET imaging with negative findings on gallium scintigraphy. Clin Nucl Med. 2005;30:193-195.

55. Prager E, Wehrschuetz M, Bisail B, et al. Comparison of ${ }^{18} \mathrm{~F}-\mathrm{FDG}$ and ${ }^{67} \mathrm{Ga}-$ citrate in sarcoidosis imaging. Nuklearmedizin. 2008;47:18-23.

56. Prabhaker HB, Rabinowitz CB, Gibbons FK, O'Donnell FJ, Shepard JA, Aquino SL. Imaging features of sarcoidosis on MDCT, FDG PET, and PET/ CT. AJR. 2008;190(suppl):S1-S6.

57. Braun JJ, Kessler R, Constantinesco A, et al. ${ }^{18}$ F-FDG PET/CT in sarcoidosis management: review and report of 20 cases. Eur J Nucl Med Mol Imaging. 2008;35:1537-1543.

58. Stange EF, Travis SP, Vermeire S, et al. European evidence based consensus on the diagnosis and management of Crohn's disease: definitions and diagnosis. Gut. 2006;55(suppl 1):i1-i15.

59. Spier BJ, Perlman SB, Reichelderfer M. FDG PET in inflammatory bowel disease. Q J Nucl Med Mol Imaging. 2009;53:64-71.

60. Molnár T, Papós M, Gyulai C, et al. Clinical value of technetium-99m-HMPAOlabeled leukocyte scintigraphy and spiral computed tomography in active Crohn's disease. Am J Gastroenterol. 2001;96:1517-1521.

61. Rispo A, Imbriaco M, Celentano L, et al. Noninvasive diagnosis of small bowel Crohn's disease: combined use of bowel sonography and Tc-99m-HMPAO leukocyte scintigraphy. Inflamm Bowel Dis. 2005;11:376-382.

62. Horsthuis K, Bipat S, Bennink RJ, Stooker J. Inflammatory bowel disease diagnosed with US, MR, scintigraphy and CT: meta-analysis of prospective studies. Radiology. 2008;247:64-78.

63. Charron M, del Rosario FJ, Kocoshis SA. Pediatric inflammatory bowel disease: assessment with scintigraphy with ${ }^{99 m} \mathrm{Tc}$ white blood cells. Radiology. 1999;212: 507-513. 
64. Bennink RJ, Peeters M, Rutgeerts P, et al. Evaluation of early treatment response and predicting the need for colectomy in active ulcerative colitis with ${ }^{99 m}$ Tc-HMPAO white blood cell scintigraphy. J Nucl Med. 2004;45: 1698-1704.

65. Lemberg DA, Issenman RM, Cawdron R, et al. Positron emission tomography in the investigation of pediatric inflammatory bowel disease. Inflamm Bowel Dis. 2005;11:733-738.

66. Löffler M, Weckesser M, Franzius C, et al. High diagnostic value of ${ }^{18}$ F-FDGPET in pediatric patients with chronic inflammatory bowel disease. Ann NY Acad Sci. 2006;1072:379-385.

67. Neurath MF, Vehling D, Schunk K, et al. Noninvasive assessment of Crohn's disease activity: a comparison of ${ }^{18} \mathrm{~F}$-fluorodeoxyglucose positron emission tomography, hydromagnetic resonance imaging, and granulocyte scintigraphy with labeled antibodies. Am J Gastroenterol. 2002;97:1978-1985.

68. Meisner RS, Spier BJ, Einarsson S, et al. Pilot study using PET/CT as a novel, noninvasive assessment of disease activity in inflammatory bowel disease. Inflamm Bowel Dis. 2007;13:993-1000.

69. Louis E, Ancion G, Colard A, et al. Noninvasive assessment of Crohn's disease intestinal lesions with ${ }^{18}$ F-FDG PET/CT. J Nucl Med. 2007;48:1053-1059.

70. Das CJ, Makharia G, Kumar R, et al. PET CT enteroclysis: a new technique for evaluation of inflammatory disease of the intestine. Eur J Nucl Med Mol Imaging. 2007;34:2106-2114.

71. Ladas SD, Triantafyllou K, Spada C, et al. European Society of Gastrointestinal Endoscopy (ESGE): recommendations (2009) on clinical use of video capsule endoscopy to investigate small-bowel, esophageal and colonic diseases. Endoscopy. 2010;42:220-227.

72. Banerjee R, Bhargav P, Reddy P, et al. Safety and efficacy of the M2A patency capsule for diagnosis of critical intestinal patency: results of a prospective clinical study. J Gastroenterol Hepatol. 2007;22:2060-2063.

73. Bleeker-Rovers CP, van der Meer JWM, Oyen WJG. Fever of unknown origin. Semin Nucl Med. 2009;39:81-87.

74. Meller J, Altenvoerde G, Munzel U, et al. Fever of unknown origin: prospective comparison of $\left[{ }^{18} \mathrm{~F}\right] \mathrm{FDG}$ imaging with a double-head coincidence camera and gallium-67 SPECT. Eur J Nucl Med. 2000;27:1617-1625.

75. Kjaer A, Lebech AM, Eigtved A, Hojgaard L. Fever of unknown origin: prospective comparison of diagnostic value of ${ }^{18} \mathrm{~F}$-FDG-PET and ${ }^{111} \mathrm{In}$-granulocyte scintigraphy. Eur J Nucl Med Mol Imaging. 2004;31:622-626.

76. Buysschaert I, Vanderschueren S, Blockmans D, et al. Contribution of 18fluorodeoxyglucose positron emission tomography to the workup of patients with fever of unknown origin. Eur J Intern Med. 2004;15:151-156.

77. Bleeker-Rovers CP, Vos FJ, Kleijn EM, et al. A prospective multi-center study of fever of unknown origin: the yield of a structured diagnostic protocol. Medicine (Baltimore). 2007;86:26-38.

78. Federici L, Blondet C, Imperiale A, et al. Value of ${ }^{18} \mathrm{~F}-\mathrm{FDG}-\mathrm{PET} / \mathrm{CT}$ in patients with fever of unknown origin and unexplained prolonged inflammatory syndrome: a single centre analysis experience. Int J Clin Pract. 2010;64:55-60.

79. Lorenzen J, Buchert R, Bohuslavizki KH. Value of FDG PET in patients with fever of unknown origin. Nucl Med Commun. 2001;22:779-783. 